\title{
REVIEWS
}

\section{Urinary Diversion Metabolic Complications - Underestimated Problem}

\author{
${ }^{1}$ Department and Clinic of Urology, Wroclaw Medical University, Poland \\ ${ }^{2}$ Department and Clinic of Nephrology and Transplantation Medicine, Wroclaw Medical University, \\ Poland
}

A - research concept and design; $\mathbf{B}$ - collection and/or assembly of data; $\mathbf{C}$ - data analysis and interpretation;

$\mathbf{D}$ - writing the article; $\mathbf{E}$ - critical revision of the article; $\mathbf{F}$ - final approval of article; $\mathbf{G}$ - other

\begin{abstract}
Bladder cancer is one of the most frequent human cancers. In 2011 more than six thousand people in Poland developed $\mathrm{BC}$ and more than three thousand died because of it. Treatment of bladder cancer depends on its stage. In less advanced tumours (Ta, Tcis, T1) transurethral resection of bladder tumor with adjuvant immunotherapy is often therapeutic. In more advanced cases $(\geq \mathrm{T} 2)$ radical cystectomy is needed. There are several surgical types of postcystectomy urinary diversion divided into two fundamental types - enabling and not enabling urine continence. The most common procedures include ureterocutaneostomy, ileal or colon conduit, orthopic ileal bladder, heterotopic continent bladder replacement (pouch) and urinary diversion via the rectum. Depending on type of cystectomy, various metabolic complications occur, because the absorptive-secretory function of used bowel segment is intact. Complications include bowel dysfunction, malabsorption of various vitamins, acid-base imbalance, electrolyte imbalance, abnormalities in bone metabolism, formation of renal calculi, secondary malignancies and disturbances in function of kidneys or liver. Early diagnosed complications can be treated easier, recognised in advanced stages are often irreversible. In our paper we present review of different approaches to bladder cancer treatment and metabolic complications occurring after these procedures (Adv Clin Exp Med 2014, 23, 4, 633-638).
\end{abstract}

Key words: bladder cancer, urinary diversion, metabolic complications.

Bladder cancer $(\mathrm{BC})$ is one of the most frequent human cancers. In 2011 more than six thousand people in Poland developed BC and more than three thousand died because of it. In terms of morbidity in 2011 it was the fifth in male and fifteenth in female cancer in Poland [1]. Frequently, the sole symptom of BC is microscopic or macroscopic haematuria, without any pain or urinary disorders. Other possible symptoms like polyuria or urinary urgency can indicate carcinoma in situ. However, palpable tumor in abdomen, bone pain or weight loss can identify advanced and metastatic cancer.

Incidence and prevalence of $\mathrm{BC}$ increase with age and exposure to environmental toxins like cigarette smoke. It is proven that smokers have 2 to 6 times grater chance of BC development [2]. Male gender, inflammation, chronic infection of bladder and previous radiotherapy are other predisposing factors.
The most common histological type of $\mathrm{BC}$ is urothelial cancer. Staging of BC is based on invasion of bladder wall, and is described within TNM (tumor, nodes, metastases) system. Treatment of BC depends on its stage. From a clinical point of view, the most important division is one, which splits into noninfiltrating lamina muscularis tumours (Ta, Tcis, T1) and those infiltrating lamina muscularis ( $\geq \mathrm{T} 2$ ). In the first group the procedure of choice is TURB (transurethral resection of bladder tumor), which is diagnostic and often therapeutic. If TURB procedure is insufficient, according to staging and clinical situation, re-TURB or adjuvant immunotherapy can be introduced. In tumours with muscular invasion the method of choice is radical cystectomy. That procedure include removal of bladder, prostatic gland, seminal vesicles, parietal peritoneum, final parts of ureters, 
and lymph nodes located in small pelvis. In women additionally uterus with appendages and front wall of vagina should be removed $[3,4]$.

Inherent part of radical cystectomy is drainage of urine. There are several surgical types of postcystectomy urinary diversion divided into two fundamental types - enabling and not enabling urine continence. In the first group there are orthopic ileal bladder, heterotopic continent bladder replacement (pouch) and urinary diversion via the rectum. The second group includes ureterocutaneostomy and ileal or colon conduit.

Choosing the appropriate one considering patient's preferences, age, and comorbidities, as well as the tumour's oncological characteristics and surgeon's experience is very important issue.

\section{Continent Orthotopic Bladder Substitution (Neobladder)}

This form of urinary diversion most closely resembles the natural bladder's function and location [5]. Most ileal reservoirs consist of 50 to $75 \mathrm{~cm}$ of terminal ileum, which is detubularized and folded in different variations to create a spherical shape. The ureters are anastomosed to the afferent tubular segment in end-to-side refluxing fashion and caudal part of the neobladder is joined with urethra. The reservoir is located in the pelvis minor and is emptied by increasing intra-abdominal pressure. Continence mechanism is preserved by patient's own sphincter [6].

Neobladder is contraindicated in patients with impaired renal and liver function and chronic inflammatory bowel disease, as well as in patients in whom urethra is affected, or in women, whose bladder neck is invaded by the tumour [7].

\section{Heterotopic Continent Bladder Replacement}

In this form of urinary diversion creation of a reservoir is required. The conditions in which this method is used include neurogenic functional and lower urinary tract disorders, congenital malformations and tumor invasion of urethra [8].

The different variants of continent catheterizable reservoirs vary according to the bowel segment (ileocecum, colon or ileum) that is used. The continence mechanism is usually provided by ileocecal valve, submucosally implanted appendix, an ileum invagination nipple, or short, tapered segments of the intestine [8]. The pouch is voided by self-catheterization through a permanent stoma. Patients qualified to this method have to be able to self-catheterize the reservoir [9].

\section{Ureterosigmoidostomy}

Ureterosigmoidostomy is a procedure in which rectosigmoid reservoir is created and the ureters are anastomosed in an antirefluxive manner into the pouch. The anal sphincter preserves both bowel and urinary control. This type of urinary diversion requires a thorough preoperative evaluation including ultrasonography and CT scans of the pelvis and abdomen, cystoscopic resection biopsy of the tumor, as well as colonoscopy. Anal sphincter function and integrity must be also assessed before the surgery. Patients with dilated ureters, completed or planned extensive pelvic irradiation, renal insufficiency, colorectal disease or incompetent anal sphincter are not candidates for this procedure [7].

\section{Ureterocutaneostomy}

The bilateral side-to-side cutaneous ureterostomy is the simplest operative procedure for urinary diversion. The ureters are detached from the bladder and then implanted separately or jointly into the skin, so that stomas are created. The urine drains into special collection bags. This technique is less invasive than the ileal conduit urinary diversion, but carries a high complication rate, especially ureteral stenoses, often requiring ureteral catheterization [5].

The conditions that may result in a need for ureterocutaneostomy are serious comorbidities and reduced life expectancy. Palliative treatment and malfunction of previous urinary diversion are also indications for this procedure. This method is also recommended in situation when the use of bowel segments should be avoided, for instance previous or intended radiotherapy of pelvis and bowel pathologies like ulcerative colitis and Crohn's disease [10].

\section{Ileal Conduit Urinary Diversion}

The ileal conduit is the most commonly used type of urinary diversion (33 to 63\%) [11]. First described by Eugene Bricker in 1950, this simple method to perform a refluxing end-to-side ureter-small bowel anastomosis has low complication rate [12]. 
Ureters are anastomosed end-to-side into the segment of ileum in a reflexive manner, which may result in reverse flow of urine back to the kidney. Creating an ileal conduit is technically simpler and the less time consuming than a continent bladder substitution system. Additionally, when an ileal conduit is formed, shorter segment of the bowel is excised [13].

Creating urinary diversion may cause common surgical complications and infections, ileus, hydronephrosis or bowel anastomosis defects may occur [14]. Additionally many metabolic changes, rarely mentioned in the literature and often underestimated by physicians, are observed. Metabolic abnormalities protrude, because the absorptive-secretory function of used bowel segment is intact [15]. Urine has prolonged contact with big area of normal bowel epithelium and exchange of various substances is possible. Additionally length of bowel is shortened and resorption area is diminished. Complications include bowel dysfunction, malabsorption of various vitamins, acid-base imbalance, electrolyte imbalance, abnormalities in bone metabolism, formation of renal calculi, and disturbances in function of kidneys or liver. Severity of metabolic complications depends on different factors. The most important agents are the length and the type of bowel segment used for urinary diversion. Each part of bowel is different from another, consequently, complication vary depending on kind of bowel used. Other significant factors are age of patient, duration of urine detention, kidney and liver function, type of performed surgery and comorbidities [5, 15-17].

\section{Bowel Abnormalities}

Many patients with urinary diversion suffer from diarrhea [18]. The main reason is reduced absorption of bile salts and fats in shortened bowel. Normal circulation (enterohepatic cycle) of bile salts is disturbed and great amounts of them reach colon causing irritations and diarrhea. Due to loss of big quantity of bile salts, fats cannot be properly emulsified and absorbed leading to chronic steatorrhea [19]. This phenomenon is positively related with length of resected segment. Other mechanism occurs when part of colon is resected. Water reabsorption and resorption of intestine alkaline content likewise is diminished. That causes acidosis and dehydration.

In cases with resected ileocecal valve diarrhea is caused by bacterial overgrow in upper part of gastro-intestinal tract and by acceleration of intestinal transit time, which disrupts digestion and absorption of food. In some cases, reconstruction of resected ileocecal valve should be performed [20]. To prevent diarrhea after urinary diversion, abundant fibber consumption should be recommended. Sometimes use of bile salts binding drug - cholestyramine is efficient, however, it can generate deficiency of fat-soluble vitamins [21]. Additionally, it is vital to recommend large fluid intake to prevent dehydration $[15,22]$.

\section{$B_{12}$ Absorption Disorders}

Resection of terminal ileum, where vitamine $B_{12}$ (cobalamin) is absorbed leads to it's hypovitaminosis. Mild hypovitaminosis is predominantly asymptomatic, however, after body supply exhaustion, it may cause megaloblastic anaemia, Hunter's glossitis, or severe neurologic injuries $[5,15,23]$. Therefore, oral supplementation should be considered in patients with urinary diversion.

\section{Renal Function}

Renal function can deteriorate after urinary diversion but the cause of this phenomenon is not completely clear. Urinary diversion is firm indication for renal function monitoring. It can be achieved by using creatinine serum levels, however, this method is not very accurate, especially in patients with low filtration rate. It was shown in literature, that estimated glomerular filtration rate (eGFR) based on creatinine serum level, is lower in patients after urinary diversion [24]. Using other agents like cystatin c, nuclear scans or single injection of ${ }^{51} \mathrm{Cr}$-EDTA for the determination of glomerular filtration rate seems to be more adequate [25].

Renal impairment may possibly occur due to recurrent infections (for which those patients are prone) [18] and/or obstruction of urinary tract caused by ureterointestinal anastomosis defects and urolithiasis.

\section{Urolithiasis}

The formation of renal calculi after urinary diversion is well documented [26]. Factors in urine, which favour stones formations, are alkaline $\mathrm{pH}$, decreased concentration of citrate and increased concentration of phosphate, calcium and sulphate. Stones are more frequently localized in upper urinary tract. Urinary stones are also formed in reservoir. This is due to the fact that the bowel epithelium promotes calculi formation itself. Some foreign materials like staples left after procedure 
are responsible for urolithiasis as well [27]. Other conductive factors are poor emptying of the reservoir and tendency of bowel epithelium exposed to the effects of urine components to infections, which, especially with urea-splitting organisms, can cause struvit (magnesium ammonium phosphate) lithiasis [28]. The most predisposed to urolithiasis type of urinary diversion is Kock pouch. Up to $43 \%$ patients with this type of pouch develop lithiasis. It is caused by often coexisting infection and abundance of foreign material used for this form of pouch creation [29]. Treatment of lithiasis is based on electroshock wave lithotripsy (ESWL) or the percutaneous endoscopy. For bigger concrements removal laparotomy and enterocystoscopy can be used. For preventing recurrent bacteriuria close surveillance and antibiotic prophylaxis is recommended [30].

\section{Acid-Base and Ions Imbalance}

Bowel part used for urinary diversion maintains its absorptive function, what enables many substances like urine, ammonia, hydrogen and chloride to be reabsorbed back to the blood flow. That results with metabolic hyperchloremic acidosis and increased load of nitrogen compounds, especially when colonic part is used for urinary diversion [27]. Reduced kidney function, which is common in patients with urinary diversion, additionally deteriorates acidosis. Majority of patients present clinically insignificant acidosis, but in some cases it can provoke bone demineralization and other complications. Treatment of acid base imbalance is based on intake of sodium citrate, sodium bicarbonate, nicotinic acid or chloropromazine [27].

Acid base imbalance and electrolyte disturbances often coexist. Electrolyte disturbances are more common in patients with continent urinary diversion. It obviously depends on type of used bowel, but hypokalemia, hypomagnesemia and hypocalcemia most often occur.

Rarely, when gastric tissue is utilized, there is no risk of acidosis, contrary hypochloremic, hypokalemic metabolic alkalosis can occur. When jejunal part is utilized hyponatremia can appear with hypochloremic acidosis. When ileum or colon is used hypokalemia with coexisting acidosis is typical $[5,31,32]$.

From ion disorders mentioned above, the most severe is hypokalemia. It can result with general weakness and arrhythmias. It can create diagnostic problems and can be taken for Guillain-Barre resembling syndrome [33].

\section{Liver Function}

In very rare cases, urinary diversion procedures can alternate liver function. Patients with liver disease are particularly prone to that complication. Increased ammonia load caused by urinary infection with urease producing bacteria and urinary obstruction can exceed liver metabolic capabilities and cause severe neurological symptoms due to hyperammonemia.[34]

\section{Drugs Metabolism}

Great part of drugs is secreted in urine as metabolised or in intact form. Drugs in patients with urinary diversion can be absorbed by bowel epithelium back to blood flow. It changes its kinetics, complicates drug therapy and increases its toxicity. These effects are documented for lithium, hydrocortisone, theophylline, phenytoin and ciprofloxacin [35]. On the other hand, drugs like methotrexate and their metabolized forms can be toxic to the bowel epithelium [36]. That phenomenon occurs with different extent and with high interindividual variability, therefore, dosage of some drugs should be adjusted to every patient separately.

\section{Bone Demineralization}

There are several probable pathomechanisms of bone demineralization. First, acidosis is buffered by carbonate mobilized from bones, calcium is unbundled into blood flow and finally secreted in urine [37]. Second, acidosis activates osteoclasts [38]. Third, acidosis undermines activation of vitamin $\mathrm{D}$ in kidneys [27]. Finally, in shortened bowel less calcium and vitamin D is reabsorbed. All that results in demineralization of bones and may cause rickets in children and osteomalacia or osteoporosis in adults. Clinical symptoms include bone pain and pathological fractures. This effect is more intensive in patients with reduced kidney function. There are none guidelines indicating when and which patient should undergo screening - densitometry. Alike to define the time, when treatment should be introduced requires further investigation. Equalizing acid base abnormalities and supplementation of vitamin C and D is recommended to treat that complication [27].

\section{Malignancies}

Some authors prove, that after urinary diversion the risk of secondary malignancy is increased. In postoperative follow-up, tumours were found in 1 to $4,5 \%$ 
of patients, additionally the risk was higher in patients with carcinogens like tobacco exposure. Gastric tissue is probably more vulnerable for secondary malignancies than other types of urinary diversion [39].

Urinary diversion through the rectum is associated with an increased risk of developing an adenocarcinoma at the site of the ureterointestinal implant. For that reason, follow-up examinations after the fifth postoperative year should include every-year colonoscopy [40].

\section{Other Complications}

A specific issue of urinary diversion using bowel segment is mucus production in the reservoir, which needs to be removed by using forced diuresis and, if required, intermittent irrigation of the reservoir by using self-catheterization [5].
If large segment of jejunum is utilized, malabsorption of nutrients can occur.

Usage of gastric tissue causes coexisting hypergastrinemia with peptic ulcer disease, iron deficiency or megaloblastic anemia [15].

\section{Conclusion}

Urinary diversion is a common procedure. It is vital that urologists and other specialists are aware and remember that beside typical surgical complications, metabolic abnormalities can develop, even though several years after procedure. Some of these complications can be managed by endoscopic procedures, but and open surgical revision is sometimes required. Early diagnosed complications can be easily treated, recognised in advanced stages are often irreversible.

\section{References}

[1] Centrum Onkologii w Warszawie. Krajowa baza danych nowotworowych. In: Warszawa 2014. http://epid.coi. waw.pl/krn/Dostęp z dnia 10.03.2014

[2] Brennan P, Bogillot O, Cordier S, Greiser E, Schill W, Vineis P, Lopez-Abente G, Tzonou A, Chang-Claude J, Bolm-Audorff U: Cigarette smoking and bladder cancer in men: a pooled analysis of 11 case-control studies. Int J Cancer 2000, 86, 289-294.

[3] Griffiths TR, Action on Bladder C: Current perspectives in bladder cancer management. Int J Clin Pract 2013, 67, 435-448.

[4] Kolodziej A, Dembowski J, Zdrojowy R, Wozniak P, Lorenz J: Treatment of high-risk superficial bladder cancer with maintenance bacille Calmette-Guerin therapy: preliminary results. BJU Int 2002, 89, 620-622.

[5] Stein R, Hohenfellner M, Pahernik S, Roth S, Thuroff JW, Rubben H: Urinary diversion - approaches and consequences. Dtsch Arztebl Int 2012, 109, 617-622.

[6] Asgari MA, Safarinejad MR, Shakhssalim N, Soleimani M, Shahabi A, Amini E: Quality of life after radical cystectomy for bladder cancer in men with an ileal conduit or continent urinary diversion: A comparative study. Urol Ann 2013, 5, 190-196.

[7] Hautmann RE, de Petriconi R, Gottfried HW, Kleinschmidt K, Mattes R, Paiss T: The ileal neobladder: complications and functional results in 363 patients after 11 years of followup. J Urol 1999, 161, 422-427; discussion 427-428.

[8] Ardelt PU, Woodhouse CR, Riedmiller H, Gerharz EW: The efferent segment in continent cutaneous urinary diversion: a comprehensive review of the literature. BJU Int 2012, 109, 288-297.

[9] Rink M, Kluth L, Eichelberg E, Fisch M, Dahlem R: Continent Catheterizable Pouches for Urinary Diversion. Eur Urol 2010, Suppl. 9, 754-762.

[10] Yoshimura K, Maekawa S, Ichioka K, Terada N, Matsuta Y, Okubo K, Arai Y: Tubeless cutaneous ureterostomy: the Toyoda method revisited. J Urol 2001, 165, 785-788.

[11] Gerharz EW: Re: Hautmann RE, Abol-Enein H, Hafez K: Urinary diversion. Urology 2007, Suppl. 69, 17-49. Urology 2008, 72, 231-232; author reply 232.

[12] Bricker EM: Bladder substitution after pelvic evisceration. Surg Clin North Am 1950, 30, 1511-1521.

[13] Madersbacher S, Schmidt J, Eberle JM, Thoeny HC, Burkhard F, Hochreiter W, Studer UE: Long-term outcome of ileal conduit diversion. J Urol 2003, 169, 985-990.

[14] Hautmann RE, Miller K, Steiner U, Wenderoth U: The ileal neobladder: 6 years of experience with more than 200 patients. J Urol 1993, 150, 40-45.

[15] Steiner MS, Morton RA: Nutritional and gastrointestinal complications of the use of bowel segments in the lower urinary tract. Urol Clin North Am 1991, 18, 743-754.

[16] McDougal WS: Metabolic complications of urinary intestinal diversion. J Urol 1992, 147, 1199-1208.

[17] Stein R, Schroder A, Thuroff JW: Bladder augmentation and urinary diversion in patients with neurogenic bladder: non-surgical considerations. J Pediatr Urol 2012, 8, 145-152.

[18] Joniau S, Benijts J, Van Kampen M, De Waele M, Ooms J, Van Cleynenbreugel B, Van Poppel H: Clinical experience with the $\mathrm{N}$-shaped ileal neobladder: assessment of complications, voiding patterns, and quality of life in our series of 58 patients. Eur Urol 2005, 47, 666-672; discussion 672-663. 
[19] Hofmann AF, Poley JR: Role of bile acid malabsorption in pathogenesis of diarrhea and steatorrhea in patients with ileal resection. I. Response to cholestyramine or replacement of dietary long chain triglyceride by medium chain triglyceride. Gastroenterology 1972, 62, 918-934.

[20] Gonzalez R: Re: Ileocecal valve reconstruction during continent urinary diversion. J Urol 1995, 153, 161-162.

[21] Jacobsen O, Hojgaard L, Hylander Moller E, Wielandt TO, Thale M, Jarnum S, Krag E: Effect of enterocoated cholestyramine on bowel habit after ileal resection: a double blind crossover study. Br Med J (Clin Res Ed) 1985, 290, 1315-1318.

[22] McDougal WS: Bladder reconstruction following cystectomy by uretero-ileo-colourethrostomy. J Urol 1986, 135, 698-701.

[23] Sagalowsky AI, Frenkel EP: Cobalamin profiles in patients after urinary diversion. J Urol 2002, 167, 1696-1700.

[24] Yang WJ, Cho KS, Rha KH, Lee HY, Chung BH, Hong SJ, Yang SC, Choi YD: Long-term effects of ileal conduit urinary diversion on upper urinary tract in bladder cancer. Urology 2006, 68, 324-327.

[25] Davidsson T, Akerlund S, White T, Olaisson G, Mansson W: Mucosal permeability of ileal and colonic reservoirs for urine. Br J Urol 1996, 78, 64-68.

[26] Terai A, Arai Y, Kawakita M, Okada Y, Yoshida O: Effect of urinary intestinal diversion on urinary risk factors for urolithiasis. J Urol 1995, 153, 37-41.

[27] Van der Aa F, Joniau S, Van Den Branden M, Van Poppel H: Metabolic changes after urinary diversion. Adv Urol 2011, 2011, 764325.

[28] Turk TM, Koleski FC, Albala DM: Incidence of urolithiasis in cystectomy patients after intestinal conduit or continent urinary diversion. World J Urol 1999, 17, 305-307.

[29] Terai A, Ueda T, Kakehi Y, Terachi T, Arai Y, Okada Y, Yoshida O: Urinary calculi as a late complication of the Indiana continent urinary diversion: comparison with the Kock pouch procedure. J Urol 1996, 155, 66-68.

[30] Cohen TD, Streem SB, Lammert G: Long-term incidence and risks for recurrent stones following contemporary management of upper tract calculi in patients with a urinary diversion. J Urol 1996, 155, 62-65.

[31] Tanrikut C, McDougal WS: Acid-base and electrolyte disorders after urinary diversion. World J Urol 2004, 22, $168-171$.

[32] Gosalbez R, Jr., Woodard JR, Broecker BH, Warshaw B: Metabolic complications of the use of stomach for urinary reconstruction. J Urol 1993, 150, 710-712.

[33] Valtier B, Mion G, Pham LH, Brochard L: Severe hypokalaemic paralysis from an unusual cause mimicking the Guillain-Barre syndrome. Intensive Care Med 1989, 15, 534-535.

[34] Albersen M, Joniau S, Van Poppel H, Cuyle PJ, Knockaert DC, Meersseman W: Urea-splitting urinary tract infection contributing to hyperammonemic encephalopathy. Nat Clin Pract Urol 2007, 4, 455-458.

[35] Ekman I, Mansson W, Nyberg L: Absorption of drugs from continent caecal reservoir for urine. Br J Urol 1989, 64, 412-416.

[36] Fossa SD, Heilo A, Bormer O: Unexpectedly high serum methotrexate levels in cystectomized bladder cancer patients with an ileal conduit treated with intermediate doses of the drug. J Urol 1990, 143, 498-501.

[37] Bettice JA, Gamble JL, Jr.: Skeletal buffering of acute metabolic acidosis. Am J Physiol 1975, 229, 1618-1624.

[38] Arnett TR, Dempster DW: Effect of pH on bone resorption by rat osteoclasts in vitro. Endocrinology 1986, 119, 119-124.

[39] Austen M, Kalble T: Secondary malignancies in different forms of urinary diversion using isolated gut. J Urol 2004, 172, 831-838.

[40] Tollefson MK, Elliott DS, Zincke H, Frank I: Long-term outcome of ureterosigmoidostomy: an analysis of patients with $>10$ years of follow-up. BJU Int 2010, 105, 860-863.

\section{Address for correspondence}

Wojciech Krajewski

Department and Clinic of Urology

Wroclaw Medical University

Borowska 213

50-556 Wrocław

Poland

Tel.: + 48717331010

E-mail: wk@softstar.pl

Conflict of interest: None declared

Received: 14.05.2014

Revised: 12.06.2014

Accepted: 1.08.2014 